\title{
Neural Networks Detect Inter-Turn Short Circuit Faults Using Inverter Switching Statistics
}

\author{
Mustafa Umit Oner, İlker Şahin, Member, IEEE, and Ozan Keysan, Member, IEEE
}

\begin{abstract}
Early detection of an inter-turn short circuit fault (ISCF) can reduce repair costs and downtime of an electrical machine. In an induction machine (IM) driven by an inverter with a model predictive control (MPC) algorithm, the controller outputs are influenced by a fault due to the fault-controller interaction. Based on this observation, this study developed a neural network model using inverter switching statistics to detect the ISCF of an IM. The method was non-invasive, and it did not require any additional sensors. In the fault detection task, the model achieved an area under receiver operating characteristics curve value of 0.9992 (95\% Confidence Interval: 0.9991 - 0.9992). At the rated operating conditions, it detected and located an ISCF of 2-turns (out of 104 turns per phase) under 0.1 seconds, a speedup of more than ten times compared to the thresholding-based method. Moreover, we published the switching vector data collected at various load torque and shaft speed values for healthy and faulty states of the IM, becoming the first publicly available ISCF detection dataset.
\end{abstract}

Index Terms-Condition monitoring, fault diagnosis, induction motor, machine learning, motor drives, multi-layer perceptron, neural networks, predictive control.

\section{INTRODUCTION}

$\mathbf{O}$ WING to its value and significance, fault diagnosis of electrical machines has been a focus of intensive research, as reflected by a plethora of publications over the past years [1]-[4]. The early detection of an incipient fault can enable repair cost and downtime reduction benefits. Furthermore, provided that the machine is fault-tolerant by design and proper identification of the inflicting fault is made, the continuum of operation with a reduced rating is also possible.

Several fault detection methods that address induction motors (IM) have been reported [5]-[7] as the IM is the most commonly used AC machine type due to its low cost and ruggedness. It is estimated that the stator faults constitute $21 \%$ of all the faults [8]. Stator faults usually start as interturn short circuit faults (ISCF) [9] and quickly develop further into complete phase-to-phase or phase-to-ground faults, which implies the total malfunctioning of the machine. Depending on the machine and the fault's structure, the time between ISCF

Mustafa Umit Oner is with the Artificial Intelligence Engineering Department, Bahcesehir University, Istanbul 34349, Turkey (e-mail: mustafaumit.oner@eng.bau.edu.tr).

illker Sahin is with the ASELSAN Inc, Ankara 06200, Turkey (e-mail: ilkersahin@aselsan.com.tr).

Ozan Keysan is with the Electrical and Electronics Engineering Department, Middle East Technical University, Ankara 06800, Turkey (email: keysan@metu.edu.tr).

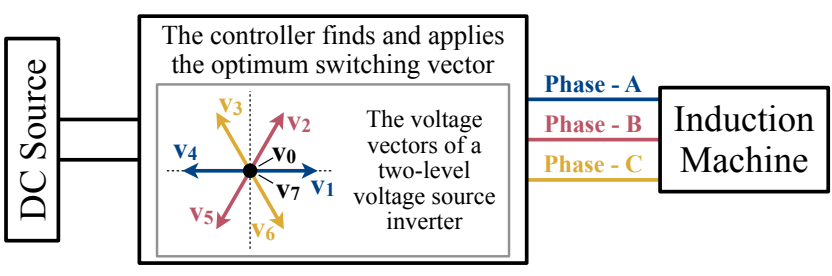

(a) Switching vectors.

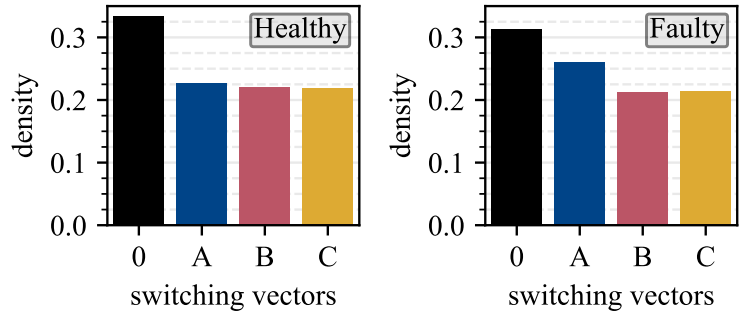

(b) Inverter switching statistics.

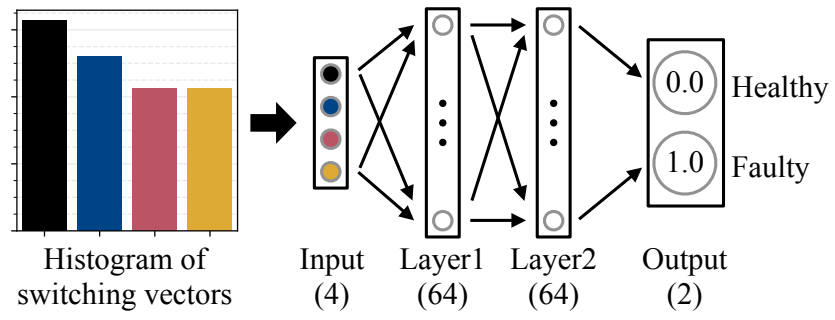

(c) Neural network model.

Fig. 1: Inverter switching statistics and neural network model. (a) The voltage vectors of a two-level voltage source inverter. The controller finds the optimum voltage vector in view of the control outcomes, and applies it at the next switching instant. (b) Histograms of switching vectors over a period for a healthy machine and a machine with inter-turn short circuit fault are given. While aggregated 0 -vectors is represented as 0 , aggregated active vectors are represented as A, B and C. (c) The neural network model is a multi-layer perceptron consisting of an input layer (with 4 nodes), two hidden layers (Layer1 and Layer2 - each with 64 nodes) and an output layer (with 2 nodes). The model takes a histogram of switching vectors at the input and predicts whether the machine is healthy or faulty at the output. 
occurrence and the total loss of insulation is in the order of seconds [10]. Therefore, a swift and effective identification of an incipient ISCF is crucial.

An important distinction regarding the fault detection studies is the control method assumed for the motor. The motor can be line-fed (uncontrolled, open-loop) or closed-loop controlled via an inverter. There exists a complex interaction between the fault and the controller [11], [12]. The controller inherently tries to negate the fault's effect. It is shown in [13] that an IM drive implemented with model predictive control (MPC) continues to exhibit perfectly balanced phase currents under an ISCF of 3-turns (out of 104 turns per phase). However, a significant unbalance is observed for the line-fed operation under the same fault condition. This example implies that most fault detection methods developed considering line-fed machines (such as motor current signature analysis) would be less effective (if not totally useless) for a high-performance control case. Therefore, it is essential to develop a fault detection method in conjunction with the main control algorithm.

Recently, the utilization of artificial intelligence (AI) techniques, such as neural networks (NN), has been gaining increasing momentum in power electronics [14]. A particular area for which the NN approach is very suitable is the fault diagnosis of electrical machines. Several studies have developed AI-based fault detection methods as reviewed in [15][18]. They mostly use stator currents or vibration signals from additional sensors to extract the fault data.

While most of these studies are for bearing fault detection [15], few are for ISCF detection. A data-driven online detection method utilizing multiple classifiers is proposed in [19]. The fault information is acquired from phase currents and voltages. ISCFs down to $2 \%$ could have been detected. A multi-layer perceptron is trained to detect ISCFs down to $0.6 \%$ in [20]. The three-phase shifts are utilized as the input data. Similarly, a Hebbian-based unsupervised neural network is reported in [21], where the phase currents are used for fault detection. While these studies [19]-[21] consider induction machines fed from AC supplies, a closed-loop controlled IM is considered in this paper, which constitutes a fundamental difference. Although an ISCF detection method detecting ISCFs down to $4.2 \%$ in a closed-loop controlled permanent magnet machine is reported in [22], no details regarding the controller structure or the controller-fault interaction are provided. An attention recurrent neural network is used which accepts phase currents and speed information as the inputs.

This study directly utilizes the switching sequences generated by the finite control-set model predictive controller (FCS-MPC) for ISCF diagnosis, therefore no additional sensor or hardware is required for fault detection purposes. The voltage vectors of a two-level voltage source inverter (2LVSI) are depicted in Fig. 1a. In the standard FCS-MPC, the controller finds the optimum voltage vector in view of the control outcomes and applies it at the next switching instant. Hence, the controller outcomes are discrete voltage vectors and convenient for statistical approaches. The switching data utilized in this paper, which correspond to the healthy operation and the ISCF case (i.e., the faulty case), are obtained from the experimental setup of [13], with a stator flux-linkage magnitude reference of $\left|\Psi_{s}\right|=0.3 \mathrm{~Wb}$. The specifications of the control approach and the experimental setup will not be revisited here as they are already described in [13] and further detailed in [23]. The ISCF condition corresponds to a shortcircuiting of 2-turns out of 104-turns in a phase winding.

A motor drive inverter with model predictive control produces an (almost) uniform distribution of active switching vectors while driving a healthy induction machine. However, an inter-turn short circuit in the stator changes the distribution of active switching vectors since the driver tries to compensate for the fault's influence for the proper operation of the motor (Fig. 1b). This observation constitutes the basis for this study. Our primary approach is to train a neural network with the switching vector data collected for healthy and faulty cases so that the trained structure can identify and locate an ISCF. The detection performance results prove the effectiveness of the proposed approach.

\section{Neural Network Based ISCF Detection}

This study designs a neural network model detecting interturn short circuit faults in an induction machine driven by an inverter with model predictive control (Fig. 1c). We formulate ISCF detection as a classification problem using inverter switching statistics.

\section{A. Problem Formulation}

Let $\boldsymbol{X}=\left\{\boldsymbol{x}_{1}, \cdots, \boldsymbol{x}_{N}\right\}$ be a set of training samples such that each sample $\boldsymbol{x}_{i} \in \mathbb{R}^{D}$ has a corresponding ground-truth label $\boldsymbol{y}_{i}=\left[y_{i}^{1}, \cdots, y_{i}^{K}\right] \in\{0,1\}^{K}$ where $\sum_{k=1}^{K} y_{i}^{k}=1$. Given our model is represented as a function parameterized by $\theta, f_{\theta}: \mathbb{R}^{D} \rightarrow \mathbb{R}^{K}$, it predicts the label of an input sample $\boldsymbol{x}_{i}$ as $\hat{\boldsymbol{y}}_{i}=f_{\theta}\left(\boldsymbol{x}_{i}\right)=\left[\hat{y}_{i}^{1}, \cdots, \hat{y}_{i}^{K}\right] \in \mathbb{R}^{K}$ such that $\hat{y}_{i}^{k} \geq 0 \forall k$ and $\sum_{k=1}^{K} \hat{y}_{i}^{k}=1$. We train our model end-to-end using categorical-cross entropy as the loss function (1).

$$
\text { loss }=\frac{1}{N} \sum_{i=1}^{N} \sum_{k=1}^{K} y_{i}^{k} \log \hat{y}_{i}^{k}
$$

\section{B. Neural Network Model Architecture}

The neural network model is a multi-layer perceptron consisting of an input layer with 4 nodes, two hidden layers with 64 nodes, and an output layer with 2 nodes (Fig. 1c). The model accepts a histogram of inverter switching statistics at the input and predicts the machine's status (healthy or faulty) at the output. Hidden layers have a ReLU activation function $(f(x)=\max (0, x))$ followed by a dropout with a rate of 0.5 . The output layer has a softmax activation function producing normalized probability values, i.e., adding up to 1 .

\section{Machine Learning Dataset}

Switching vectors of an inverter with model predictive control were collected for different conditions of an induction machine in an experimental setup explained in [13], and also further detailed in [23]. The induction machine had a rated speed of $w=3000 \mathrm{rpm}$ and a rated torque of $T=1.20$ $\mathrm{N} \cdot \mathrm{m}$. The switching vectors were recorded as data series for 
TABLE I: Data for ISCF Detection. Inverter switching vectors were collected for different conditions of an induction machine with a rated speed of $w=3000 \mathrm{rpm}$ and a rated torque of $T=1.20 \mathrm{~N} \cdot \mathrm{m}$. For each data series, speed $(w)$, torque $(T)$, measured electrical frequency $\left(f_{e}\right)$, and machine status as "Healthy" or "Faulty" are presented. Each data series in the first part was divided into two such that the first $70 \%$ and the remaining $30 \%$ were used to create samples for the training and validation sets, respectively. Data series in the second part were used to create samples for the test set.

\begin{tabular}{|c|c|c|c|c|c|c|c|c|c|}
\hline \multicolumn{5}{|c|}{ Training and Validation } & \multicolumn{5}{|l|}{ Test } \\
\hline id & $w(\mathrm{rpm})$ & $T(\mathrm{~N} \cdot \mathrm{m})$ & $f_{e}(\mathrm{~Hz})$ & Machine Status & id & $w(\mathrm{rpm})$ & $T(\mathrm{~N} \cdot \mathrm{m})$ & $f_{e}(\mathrm{~Hz})$ & Machine Status \\
\hline 001 & 1500 & 0.30 & 25.5 & Healthy & 035 & 1500 & 0.30 & 25.5 & Healthy \\
\hline 002 & 1500 & 0.30 & 25.5 & Faulty & 036 & 1500 & 0.30 & 25.5 & Faulty \\
\hline 003 & 1500 & 0.90 & 28.0 & Healthy & 037 & 1500 & 0.90 & 28.0 & Healthy \\
\hline 004 & 1500 & 0.90 & 28.0 & Faulty & 038 & 1500 & 0.90 & 28.0 & Faulty \\
\hline 005 & 2250 & 0.30 & 38.1 & Healthy & 039 & 2250 & 0.30 & 38.1 & Healthy \\
\hline 006 & 2250 & 0.30 & 38.1 & Healthy & 040 & 2250 & 0.30 & 38.1 & Faulty \\
\hline 007 & 2250 & 0.30 & 38.1 & Faulty & 041 & 2250 & 0.30 & 38.1 & Faulty \\
\hline 008 & 2250 & 0.30 & 38.1 & Faulty & 042 & 2250 & 1.25 & 42.0 & Healthy \\
\hline 009 & 2250 & 1.25 & 42.0 & Healthy & 043 & 2250 & 1.25 & 42.0 & Healthy \\
\hline 010 & 2250 & 1.25 & 42.0 & Faulty & 044 & 2250 & 1.25 & 42.0 & Faulty \\
\hline 011 & 2250 & 1.25 & 42.0 & Faulty & 045 & 2250 & 1.25 & 42.0 & Faulty \\
\hline 012 & 3000 & 0.30 & 50.6 & Healthy & 046 & 3000 & 0.30 & 50.6 & Healthy \\
\hline 013 & 3000 & 0.30 & 50.6 & Healthy & 047 & 3000 & 0.30 & 50.6 & Healthy \\
\hline 014 & 3000 & 0.30 & 50.6 & Faulty & 048 & 3000 & 0.30 & 50.6 & Faulty \\
\hline 015 & 3000 & 0.30 & 50.6 & Faulty & 049 & 3000 & 0.30 & 50.6 & Faulty \\
\hline 016 & 3000 & 1.20 & 54.4 & Healthy & 050 & 3000 & 1.20 & 54.4 & Healthy \\
\hline 017 & 3000 & 1.30 & 54.8 & Faulty & 051 & 3000 & 1.30 & 54.8 & Faulty \\
\hline 018 & 3000 & 1.35 & 55.3 & Healthy & 052 & 3000 & 1.35 & 55.3 & Healthy \\
\hline 019 & 3000 & 1.35 & 55.3 & Faulty & 053 & 3000 & 1.35 & 55.3 & Faulty \\
\hline 020 & 3750 & 0.30 & 63.1 & Healthy & 054 & 3750 & 0.30 & 63.1 & Healthy \\
\hline 021 & 3750 & 0.30 & 63.1 & Healthy & 055 & 3750 & 0.30 & 63.1 & Faulty \\
\hline 022 & 3750 & 0.30 & 63.1 & Faulty & 056 & 3750 & 0.30 & 63.1 & Faulty \\
\hline 023 & 3750 & 0.30 & 63.1 & Faulty & 057 & 3750 & 1.25 & 67.5 & Healthy \\
\hline 024 & 3750 & 1.25 & 67.5 & Healthy & 058 & 3750 & 1.25 & 67.5 & Healthy \\
\hline 025 & 3750 & 1.25 & 67.5 & Faulty & 059 & 3750 & 1.25 & 67.5 & Faulty \\
\hline 026 & 3750 & 1.25 & 67.5 & Faulty & 060 & 3750 & 1.25 & 67.5 & Faulty \\
\hline 027 & 4500 & 0.30 & 75.6 & Healthy & 061 & 4500 & 0.30 & 75.6 & Healthy \\
\hline 028 & 4500 & 0.30 & 75.6 & Healthy & 062 & 4500 & 0.30 & 75.6 & Faulty \\
\hline 029 & 4500 & 0.30 & 75.6 & Faulty & 063 & 4500 & 1.15 & 79.8 & Healthy \\
\hline 030 & 4500 & 0.30 & 75.6 & Faulty & 064 & 4500 & 1.15 & 79.8 & Faulty \\
\hline 031 & 4500 & 1.15 & 79.8 & Healthy & & & & & \\
\hline 032 & 4500 & 1.15 & 79.8 & Healthy & & & & & \\
\hline 033 & 4500 & 1.15 & 79.8 & Faulty & & & & & \\
\hline 034 & 4500 & 1.15 & 79.8 & Faulty & & & & & \\
\hline
\end{tabular}

the induction machine operating at different combinations of speed and torque at healthy and faulty states (see Table I). Each data series consisted of 22000 switching vectors. Interturn short circuit fault was introduced over 2 turns (out of 104 turns) in one phase of the machine (see [13] for details).

Collected data series were segregated into two sets such that the first set was for training and validation, and the second set was for the test. Each data series in the first set was further split into two data series. The first $70 \%$ of data points constituted a data series for training, and the remaining $30 \%$ constituted a data series for validation. Then, machine learning datasets of training, validation, and test were prepared by creating sample and label pairs over respective data series.

Over a data series, multiple samples were created in a sliding window fashion with a step size of one. A sample was created by calculating the histogram of switching vectors over a window of five electrical periods $(\approx 92 \mathrm{~ms}$ at the rated speed and torque) (Fig. 1b, see supplementary Fig. S1, Fig. S2, and Fig. S3 for the histograms over the data series in the training, validation, and test sets). Note that switching vectors were aggregated as 0 -vectors $\left(v_{0}-v_{7}\right)$, phase $\mathrm{A}$ vectors $\left(v_{1}-v_{4}\right)$, phase $\mathrm{B}$ vectors $\left(v_{2}-v_{5}\right)$, and phase $\mathrm{C}$ vectors $\left(v_{3}-v_{6}\right)$. The machine's status (i.e., healthy or faulty) was assigned as the sample's label.

\section{Training and Testing of The NN Model}

The neural network model was trained on samples created from training data series using the loss function given in Eq. 1. Early stopping based on loss in the validation dataset was employed to avoid overfitting. Finally, the model's performance was evaluated on the unseen test dataset.

The area under the receiver operating characteristic curve (AUROC) was used as the performance metric. We also calculated the 95\% confidence interval (CI) of the AUROC using the percentile bootstrap method [24].

\section{E. Data and Code Availability}

The data and code have been deposited at IEEE DataPort under the https://doi.org/10.21227/chp0-5x97 [25].

\section{EXPERIMENTS}

\section{A. The NN Detects ISCF}

We checked the performance of the neural network model on ISCF detection. For each sample in the test dataset, we 


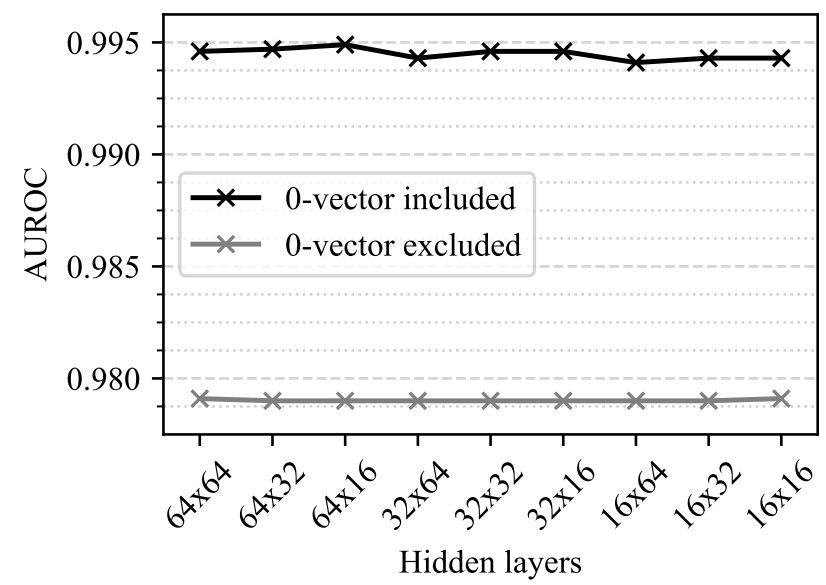

Fig. 2: The neural network models detect ISCF. Different NN models using the same architecture with different number of nodes in the hidden layers were trained and tested on the ISCF detection task. The area under receiver operating characteristics curve (AUROC) calculated on the test set was used as performance metric. We trained and tested the models using histogram of all vectors ( 0 -vector included) and active vectors only ( 0 -vector excluded).

obtained a prediction from the trained model and plotted the receiver operating characteristic curve. We got an AUROC of 0.9992 (95\% CI: $0.9991-0.9992$ ).

We checked the effect of the model's capacity on the model's performance by varying the hidden layer sizes. As expected, we observed that the model's performance decreased with decreasing model capacity (Fig. 2). Nevertheless, the performance decrease was not drastic. Hence, smaller networks could be preferable for real-world deployment since they require less computational power.

Besides, we checked the model's performance on identifying if a data series was collected at the healthy or faulty state of the machine. Prediction for a data series was obtained as the average of predictions of all samples created from the data series. Although we observed wrong predictions at the sample level in the few data series, the model perfectly identified the machine's status at the data series level (Fig. 3, supplementary Fig. S4, and Fig. S5).

\section{B. O-vectors Help The NN Detect ISCF}

We know that the proportion of 0 -vectors provides information about the speed and torque of the machine, which can be valuable for the model. To test the effect of 0 -vectors on the performance of the neural network model, we excluded 0 -vectors from histogram calculation and reran our experiments. As expected, we observed a performance decrease (Fig. 2). Hence, we concluded that 0 -vectors helped the neural network models detect ISCF.

Furthermore, we observed a decrease in fault detection performance for the speed values beyond $3750 \mathrm{rpm}$ (Fig. 3). This region corresponds to the verge of overmodulation in an inverter control system with a carrier-based modulation, where the percentage of zero vectors significantly decreases.

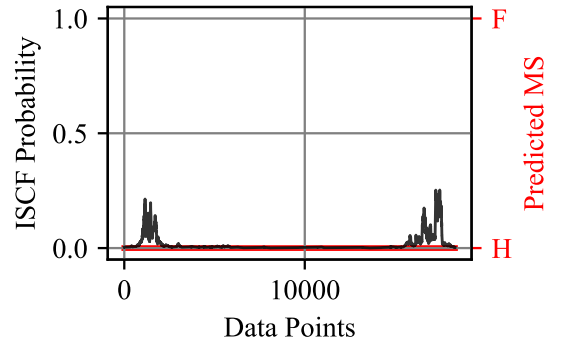

(a) id=050, $w=3000, T=1.20, \mathrm{MS}=\mathrm{H}$

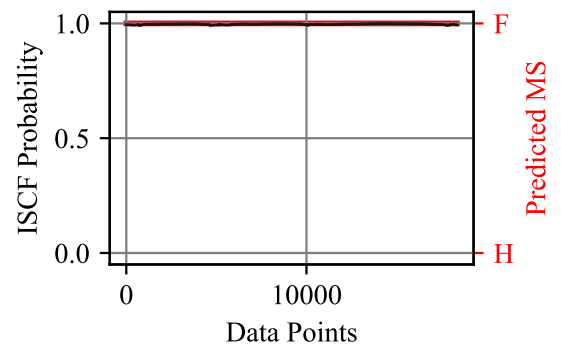

(b) id=051, $w=3000, T=1.30, \mathrm{MS}=\mathrm{F}$

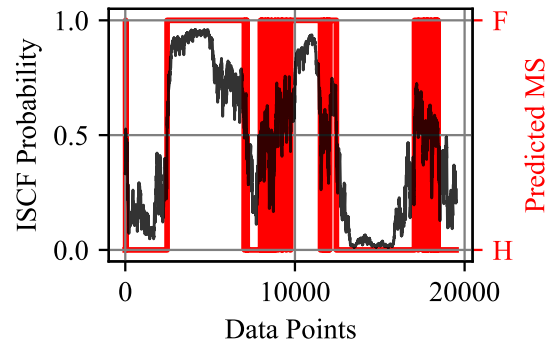

(c) id=063, $w=4500, T=1.15, \mathrm{MS}=\mathrm{H}$

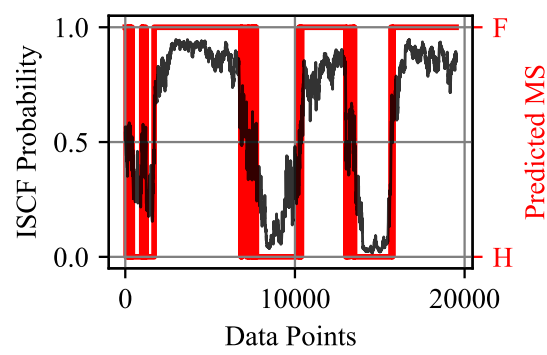

(d) id=064, $w=4500, T=1.15, \mathrm{MS}=\mathrm{F}$

Fig. 3: Sample-level ISCF predictions on the data series in the test dataset. At each point of a data series (i.e., for a sample), inter-turn short circuit fault (ISCF) probability was obtained from the trained model. ISCF probability and predicted machine status (MS) obtained by thresholding the predicted probability value with 0.5 are presented for four data series having wrong predictions. For each data series, id, speed (rpm), torque (N.m), and MS ((H)ealthy, (F)aulty) are also given. See supplementary Fig. S4 and Fig. S5 for all data series. 


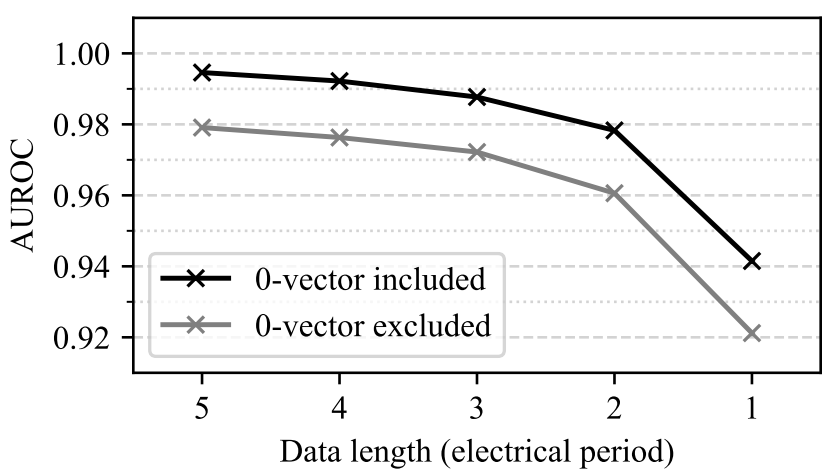

Fig. 4: Statistics over longer intervals improve performance. Switching vector histograms were calculated over an interval of multiples of electrical frequency $(5,4,3,2$, and 1) while training the NN models. We compared the effect of interval length on the model's performance using AUROC on the test set both for 0 -vector included and 0 -vector excluded cases.

The switching vector statistics were also not as responsive to the occurrence of the fault as they were at the slower speeds.

\section{Statistics Over Longer Intervals Improve Performance}

We trained and tested our models with sample and label pairs. A sample was created by calculating the histogram of switching vectors over a window of five electrical periods $(\approx 92 \mathrm{~ms}$ at the rated speed and torque). In an ideal system (where there is no noise), an ISCF can be detected using inverter switching statistics calculated over one electrical period. However, the system is noisy in practice, and the noise directly affects the model's performance.

We investigated the effects of the interval length, over which switching vector histograms were calculated, on the performance of neural network models. We observed that as the length of the interval decreased (multiples of electrical period: 5, 4, 3, 2, and 1), the model's performance also decreased (Fig. 4). In short, we concluded that statistics over longer intervals improved the model's performance.

One interesting observation was that the performance decay was gradual from five to two periods. Nevertheless, it was drastic from two periods to a single period. Our observation was also consistent with the findings of [13], which uses a thresholding-based method over switching vector statistics. Besides, the contribution of 0-vectors in the model's performance was evident (Fig. 4).

\section{The NN Identifies Faulty Phase in a Machine with ISCF}

After we showed that a neural network model successfully detected ISCF, we also checked if it could identify the faulty phase. We prepared a small dataset using data series collected at the rated speed and around the rated torque of the induction machine (Table II).

We modified the neural network architecture used in ISCF detection to a multi-class classification model with four classes corresponding to the machine's status of healthy, faulty (A),
TABLE II: Data for Faulty Phase Detection. For each data series, speed $(w)$, torque $(T)$, measured electrical frequency $\left(f_{e}\right)$, and machine status as "Healthy" or "Faulty" with phase info indicated in parentheses $(\mathrm{A} / \mathrm{B} / \mathrm{C})$ are presented. Each data series in the top part was divided into two such that the first $70 \%$ and the remaining $30 \%$ were used to create samples for the training and validation sets, respectively. Data series in the bottom part were used to create samples for the test set.

\begin{tabular}{lllll}
\hline \multicolumn{5}{l}{ Training and Validation } \\
\hline id & $w(\mathrm{rpm})$ & $T(\mathrm{~N} \cdot \mathrm{m})$ & $f_{e}(\mathrm{~Hz})$ & Machine Status \\
\hline 016 & 3000 & 1.20 & 54.4 & Healthy \\
018 & 3000 & 1.35 & 55.3 & Healthy \\
017 & 3000 & 1.30 & 54.8 & Faulty (A) \\
065 & 3000 & 1.25 & 54.6 & Faulty (B) \\
066 & 3000 & 1.25 & 54.6 & Faulty (C) \\
\hline \hline Test & & & \\
\hline id & $w(\mathrm{rpm})$ & $T(\mathrm{~N} \cdot \mathrm{m})$ & $f_{e}(\mathrm{~Hz})$ & Machine Status \\
\hline 050 & 3000 & 1.20 & 54.4 & Healthy \\
052 & 3000 & 1.35 & 55.3 & Healthy \\
051 & 3000 & 1.30 & 54.8 & Faulty (A) \\
067 & 3000 & 1.25 & 54.6 & Faulty (B) \\
068 & 3000 & 1.25 & 54.6 & Faulty (C) \\
\hline
\end{tabular}

TABLE III: Faulty phase detection. The same NN architecture was modified to a multi-class classification model detecting faulty phase as well. The confusion matrix obtained on the test set is presented.

\begin{tabular}{llllll}
\hline & \multicolumn{4}{c}{ Predicted } \\
\cline { 3 - 6 } & & Healthy & Faulty (A) & Faulty (B) & Faulty (C) \\
\hline \multirow{4}{*}{ Truth } & Healthy & 1.000 & 0.000 & 0.000 & 0.000 \\
& Faulty (A) & 0.000 & 1.000 & 0.000 & 0.000 \\
& Faulty (B) & 0.000 & 0.000 & 1.000 & 0.000 \\
& Faulty (C) & 0.003 & 0.000 & 0.000 & 0.997 \\
\hline
\end{tabular}

faulty (B), and faulty (C). Then, we trained the model on the training set with early stopping based on loss in the validation set and evaluated its performance on the test set. The model successfully detected ISCF and identified the faulty phase (Table III). It achieved an accuracy of 0.9995. As in the ISCF detection task, our neural network model perfectly detected ISCF and identified the faulty phase at the data series level.

\section{E. The NN Outperforms Thresholding Based Method}

The duration of high electrical currents passing through the shorted turns during an ISCF is critical for the repair and possible fault-tolerant operation of the machine. As ISCF detection time takes longer, the chance of successfully repairing and running the machine gets lower. Therefore, we compared the performance of our neural network model with the thresholding-based method of [13] in terms of ISCF detection time. At various load torque and shaft speed values, while the thresholding-based method detected ISCF in between 0.5 to 2 seconds, the neural network model detected ISCF in between 0.074 to 0.196 seconds. There was a speedup of more than ten times at the rated operating conditions $(\approx 1$ second for the thresholding-based method and 0.092 seconds for our neural network model). 


\section{CONCLUSION}

Early detection of an ISCF in an electrical machine is vital for its maintenance. This study developed a neural network model that detects an ISCF in an IM driven by an inverter with an MPC algorithm. The model accepted the histogram of inverter switching vectors, which are readily available, as input and predicted the machine's status (healthy or faulty) at the output (Fig. 1). An ISCF in the IM was successfully detected under 0.1 seconds with almost perfect performance (Fig. 2). Besides, the faulty phase was identified with an accuracy of 0.9995 (Table III).

In our experiments, while the largest network (with two hidden layers of 64 nodes) performed the best in the ISCF detection task, the performance of the smallest network (with two hidden layers of 16 nodes) was also good enough for realworld deployment (Fig. 2). Moreover, it requires less memory and computational resources, facilitating its deployment in the same processor alongside the controller algorithm.

Our experiments validated that 0 -vectors contained valuable information for ISCF detection (Fig. 2), and statistics over longer intervals improved the performance (Fig. 4). Nevertheless, there was a trade-off between better performance and faster ISCF detection in determining the optimum interval for statistics calculation. We concluded that an interval of three to five electrical periods was reasonable.

Lastly, we observed that the model's performance started to degrade beyond the rated speed and torque values (Fig. 3), which corresponds to the operation on the verge of overmodulation, where the utilization of zero vectors significantly decreases. This could be due to the limited available data around these operation regions of the IM. We had around 300k samples in our training set; however, they were created from only 26 independent data series (Table I). Since data collection is quite expensive, our dataset was minimal compared to traditional deep learning datasets containing millions of independent samples [26].

Hence, the collection of an extensive dataset with broad coverage of the IM's operation regions and the real-world deployment of our ISCF detection models are reserved for future work.

\section{ACKNOWLEDGMENT}

We thank Öztürk Şahin Alemdar for constructive comments on the manuscript.

\section{REFERENCES}

[1] S. Nandi, H. Toliyat, and X. Li, "Condition monitoring and fault diagnosis of electrical motors-a review," IEEE Transactions on Energy Conversion, vol. 20, DOI 10.1109/TEC.2005.847955, no. 4, pp. 719729, 2005.

[2] H. Henao, G.-A. Capolino, M. Fernandez-Cabanas, F. Filippetti, C. Bruzzese, E. Strangas, R. Pusca, J. Estima, M. Riera-Guasp, and S. Hedayati-Kia, "Trends in fault diagnosis for electrical machines: A review of diagnostic techniques," IEEE Industrial Electronics Magazine, vol. 8, DOI 10.1109/MIE.2013.2287651, no. 2, pp. 31-42, 2014.

[3] A. Gandhi, T. Corrigan, and L. Parsa, "Recent advances in modeling and online detection of stator interturn faults in electrical motors," IEEE Transactions on Industrial Electronics, vol. 58, DOI 10.1109/TIE.2010.2089937, no. 5, pp. 1564-1575, 2011.
[4] Y. Chen, S. Liang, W. Li, H. Liang, and C. Wang, "Faults and diagnosis methods of permanent magnet synchronous motors: A review," Applied Sciences, vol. 9, no. 10, p. 2116, 2019.

[5] A. Bellini, F. Filippetti, C. Tassoni, and G.-A. Capolino, "Advances in diagnostic techniques for induction machines," IEEE Transactions on Industrial Electronics, vol. 55, DOI 10.1109/TIE.2008.2007527, no. 12, pp. 4109-4126, 2008.

[6] A. Siddique, G. Yadava, and B. Singh, "A review of stator fault monitoring techniques of induction motors," IEEE Transactions on Energy Conversion, vol. 20, DOI 10.1109/TEC.2004.837304, no. 1, pp. 106-114, 2005.

[7] R. T. Purushottam Gangsar, "Signal based condition monitoring techniques for fault detection and diagnosis of induction motors: A stateof-the-art review," Mechanical Systems and Signal Processing, vol. 144, DOI 10.1016/j.ymssp.2020.106908, 2020.

[8] A. H. Bonnett and C. Yung, "Increased efficiency versus increased reliability," IEEE Industry Applications Magazine, vol. 14, DOI 10.1109/MIA.2007.909802, no. 1, pp. 29-36, 2008.

[9] A. Bonnett and G. Soukup, "Cause and analysis of stator and rotor failures in three-phase squirrel-cage induction motors," IEEE Transactions on Industry Applications, vol. 28, DOI 10.1109/28.148460, no. 4, pp. 921-937, 1992.

[10] C. Gerada, K. Bradley, M. Sumner, P. Wheeler, S. Picker, J. Clare, C. Whitley, and G. Towers, "The results do mesh," IEEE Industry Applications Magazine, vol. 13, DOI 10.1109/MIA.2007.322274, no. 2, pp. 62-72, 2007.

[11] S. Cheng, P. Zhang, and T. G. Habetler, "An impedance identification approach to sensitive detection and location of stator turn-to-turn faults in a closed-loop multiple-motor drive," IEEE Transactions on Industrial Electronics, vol. 58, DOI 10.1109/TIE.2010.2064276, no. 5, pp. 15451554, 2011.

[12] M. Zafarani, E. Bostanci, Y. Qi, T. Goktas, and B. Akin, "Interturn short-circuit faults in permanent magnet synchronous machines: An extended review and comprehensive analysis," IEEE Journal of Emerging and Selected Topics in Power Electronics, vol. 6, DOI 10.1109/JESTPE.2018.2811538, no. 4, pp. 2173-2191, 2018.

[13] İ. Şahin and O. Keysan, "Model predictive controller utilized as an observer for inter-turn short circuit detection in induction motors," IEEE Transactions on Energy Conversion, vol. 36, DOI 10.1109/TEC.2020.3048071, no. 2, pp. 1449-1458, 2021.

[14] B. K. Bose, "Artificial intelligence techniques: How can it solve problems in power electronics?: An advancing frontier," IEEE Power Electronics Magazine, vol. 7, DOI 10.1109/MPEL.2020.3033607, no. 4, pp. 19-27, 2020.

[15] S. Zhang, S. Zhang, B. Wang, and T. G. Habetler, "Deep learning algorithms for bearing fault diagnostics-a comprehensive review," IEEE Access, vol. 8, DOI 10.1109/ACCESS.2020.2972859, pp. $29857-$ $29881,2020$.

[16] M. Seera, C. P. Lim, S. Nahavandi, and C. K. Loo, "Condition monitoring of induction motors: A review and an application of an ensemble of hybrid intelligent models," Expert Systems with Applications, vol. 41, no. 10, pp. 4891-4903, 2014.

[17] W. Lang, Y. Hu, C. Gong, X. Zhang, H. Xu, and J. Deng, "Artificial intelligence-based technique for fault detection and diagnosis of ev motors: A review," IEEE Transactions on Transportation Electrification, DOI 10.1109/TTE.2021.3110318, pp. 1-1, 2021.

[18] L. Wen, X. Li, L. Gao, and Y. Zhang, "A new convolutional neural network-based data-driven fault diagnosis method," IEEE Transactions on Industrial Electronics, vol. 65, DOI 10.1109/TIE.2017.2774777, no. 7, pp. 5990-5998, 2018.

[19] Z. Xu, C. Hu, F. Yang, S.-H. Kuo, C.-K. Goh, A. Gupta, and S. Nadarajan, "Data-driven inter-turn short circuit fault detection in induction machines," IEEE Access, vol. 5, DOI 10.1109/ACCESS.2017.2764474, pp. $25055-25068,2017$.

[20] M. B. K. Bouzid, G. Champenois, N. M. Bellaaj, L. Signac, and K. Jelassi, "An effective neural approach for the automatic location of stator interturn faults in induction motor," IEEE Transactions on Industrial Electronics, vol. 55, DOI 10.1109/TIE.2008.2004667, no. 12, pp. 4277-4289, 2008.

[21] J. F. Martins, V. Ferno Pires, and A. J. Pires, "Unsupervised neuralnetwork-based algorithm for an on-line diagnosis of three-phase induction motor stator fault," IEEE Transactions on Industrial Electronics, vol. 54, DOI 10.1109/TIE.2006.888790, no. 1, pp. 259-264, 2007.

[22] H. Lee, H. Jeong, G. Koo, J. Ban, and S. W. Kim, "Attention recurrent neural network-based severity estimation method for interturn short-circuit fault in permanent magnet synchronous ma- 
chines," IEEE Transactions on Industrial Electronics, vol. 68, DOI 10.1109/TIE.2020.2978690, no. 4, pp. 3445-3453, 2021.

[23] İ. Şahin, "Model predictive torque control of an induction motor enhanced with an inter-turn short circuit fault detection feature," Ph.D. dissertation, Middle East Technical University, 2021.

[24] B. Efron, "Bootstrap methods: another look at the jackknife," in Breakthroughs in statistics, pp. 569-593. Springer, 1992.

[25] M. U. Oner, İ. Şahin, and O. Keysan, "Inverter switching vectors data of an fcs-mpc driven induction machine for healthy and faulty conditions," 2022. [Online]. Available: https://dx.doi.org/10.21227/chp0-5x97

[26] J. Deng, W. Dong, R. Socher, L.-J. Li, K. Li, and L. FeiFei, "Imagenet: A large-scale hierarchical image database," in 2009 IEEE Conference on Computer Vision and Pattern Recognition, DOI 10.1109/CVPR.2009.5206848, pp. 248-255, 2009.

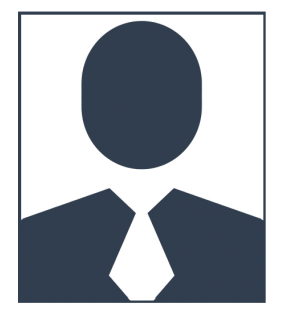

Mustafa Umit Oner got his B.S. degree in 2013 and M.S. degree in 2016 from the Electrical and Electronics Engineering Department at the Middle East Technical University, Ankara, Turkey. Then, he got his Ph.D. degree in 2021 from the Computer Science Department at the National University of Singapore, Singapore. He continues his research at the Bahcesehir University, Istanbul, Turkey. He is interested in developing novel machine learning models and machine learning-based information systems for digital histopathology and integrative multi-omics to support diagnostic and therapeutic decision-making in cancer.

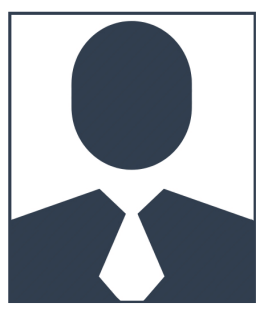

Illker Şahin (Member, IEEE) received the B.Sc., M.Sc., and Ph.D. degrees in electrical and electronics engineering, in 2010, 2014, and 2021 respectively, from Middle East Technical University (METU), Ankara, Turkey. From 2011 to 2020, he was a Teaching Assistant with METU, then he joined Aselsan in 2020, as a Lead Design Engineer. His current research interests include high-performance motor drives, predictive control, and fault diagnosis.

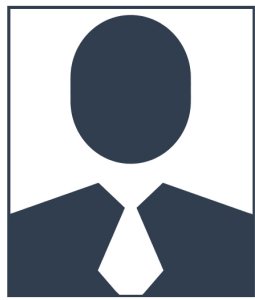

Ozan Keysan (Member, IEEE) received the master's degree from Middle East Technical University (METU), Ankara, Turkey, in 2008, and the Ph.D. degree from the University of Edinburgh, Edinburgh, Scotland, in 2014. He is currently an Associate Professor with the Electrical and Electronics Engineering Department, METU. His current research interests include renewable energy, design, and optimization of electrical machines, smart grids, superconducting machines, and permanent-magnet machines. 